\title{
Analytic bootstrap for logarithmic CFT
}

\author{
Pinaki Banerjee ${ }^{a, b}$ and Parijat Dey ${ }^{c}$ \\ ${ }^{a}$ International Centre for Theoretical Sciences, Tata Institute of Fundamental Research, \\ Shivakote, Bengaluru 560 089, India \\ ${ }^{b}$ Indian Institute of Technology Kanpur, \\ Kalyanpur, Kanpur 208016, India \\ ${ }^{c}$ Department of Physics and Astronomy, Uppsala University, \\ Box 516, SE-751 20 Uppsala, Sweden \\ E-mail: pinakib@iitk.ac.in, parijat.dey@physics.uu.se
}

ABSTRACT: We study logarithmic conformal field theory (LogCFT) in four dimensions using conformal bootstrap techniques in the large spin limit. We focus on the constraints imposed by conformal symmetry on the four point function of certain logarithmic scalar operators and compute the leading correction to the anomalous dimension of double trace operators in the large spin limit. There exist certain holographic duals to such LogCFTs, which involve higher derivative equations of motion. The anomalous dimension is related to the binding energy of a state where two scalars rotate around each other with a large angular momentum. We compute this energy shift and compare it to the anomalous dimension of the large spin double trace operators due to stress tensor exchange in the LogCFT. Our result shows that the cluster decomposition principle is satisfied for LogCFTs as long as the dimensions of the operators are positive.

KEYwords: Conformal Field Theory, Gauge-gravity correspondence

ARXIV EPRINT: 1908.10437 


\section{Contents}

1 Introduction 1

2 Logarithmic generalized free fields 3

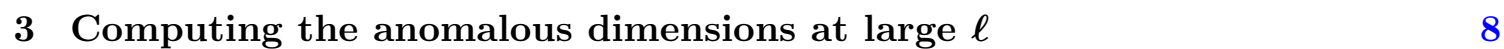

4 The dual gravity picture 10

$\begin{array}{lll}5 & \text { Conclusion } & 15\end{array}$

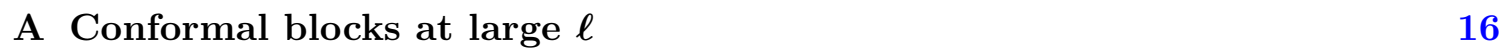

$\begin{array}{ll}\text { B Some details of the bulk computation } & 17\end{array}$

\section{Introduction}

Conformal field theories (CFTs) are quite essential in different branches of physics - particularly in statistical mechanics, condensed matter theory and string theories. They also play an important role in our present day understanding of quantum field theories. Quantum field theories can be thought of as conformal field theories deformed by some relevant perturbations. In that sense CFTs are very special points in the space of all quantum field theories. CFTs appear in physical systems when there is no characteristic length scale. Therefore the correlation functions can only have power laws. It was pointed out in the early 90's [1-3] that the structure of general CFTs allows the presence of multiplicative logarithms in correlation functions even at an RG fixed point. Such theories are called logarithmic conformal field theories (LogCFTs). Since CFTs come with no length/mass scale, one may wonder how there can be logarithms in their correlation functions. The answer lies on non-diagonalizable action of some operators of the type of a Hamiltonian. Let's see via an illustrative toy example how logarithms can appear in a CFT. Suppose the dilatation operator $\mathcal{L}_{0} \sim x \frac{\partial}{\partial x}$ acts non-diagonally on a two component scalar $\mathbb{G}$,

$$
\mathcal{L}_{0} \mathbb{G}=\left[\begin{array}{ll}
\Delta & 0 \\
1 & \Delta
\end{array}\right]\left[\begin{array}{l}
g_{1}(x) \\
g_{2}(x)
\end{array}\right]
$$

where $\mathcal{L}_{0}=\left[x \frac{\partial}{\partial x}, x \frac{\partial}{\partial x}\right]$ and $\mathbb{G}=\left[\begin{array}{l}g_{1}(x) \\ g_{2}(x)\end{array}\right]$. From this non-diagonal action of $\mathcal{L}_{0}$ we get the following equations,

$$
\begin{aligned}
& x g_{1}^{\prime}(x)=\Delta g_{1}(x) \\
& x g_{2}^{\prime}(x)=\Delta g_{2}(x)+g_{1}(x) .
\end{aligned}
$$


These are two first order differential equations with the following solutions,

$$
\begin{aligned}
& g_{1}(x)=B x^{\Delta} \\
& g_{2}(x)=A x^{\Delta}+B x^{\Delta} \log (x) .
\end{aligned}
$$

From representation theory perspective these are irreducible but indecomposable representation of conformal group. This implies that the transformation of a two component scalar of dimension $\Delta$ involves a logarithm in the correlation function. In general there can be logarithmic multiplets of rank $r \geq 1$ which can be built by acting on $r$ primary states $\left|\mathcal{O}_{i}\right\rangle$ for $i=1, \cdots r$ obeying the highest-weight condition,

$$
K_{\mu}\left|\mathcal{O}_{i}\right\rangle=0
$$

where $K_{\mu}$ is the generator for the special conformal transformation. Hence a LogCFT consists of $r$-primaries and all their descendants. However, LogCFTs are less constrained than unitary rational CFTs. In [4] Cardy took a complementary approach considering LogCFTs as limits of ordinary, non-logarithmic CFTs, whose physical interpretation is already well understood, as a parameter is taken to a particular value. In this way the properties of the corresponding LogCFTs can be derived and we are able to understand exactly where the logarithms should appear in the physical observables. While that approach was quite general, it was illustrated with several examples of physical interest, including quenched random magnets, self-avoiding walks, and percolation which makes it evident that LogCFTs are not merely mathematical artifact. One of the earliest physical systems LogCFTs made its appearance was in the context of polymers and percolation $[2,5]$. Several works has been done in other statistical mechanical systems e.g. 2D turbulence [6-8], and the abelian sand-pile model [9-11]. There are also applications in quantum condensed matter systems like disordered $[12,13]$ and the quantum Hall systems [14-17]. LogCFTs have also been studied in the context of worldsheet string theory [18-23] and AdS/CFT correspondence [24-28]. Recently Witten [29] found the necessity for LogCFTs in a first principle string theory derivation of entanglement entropy.

Although the existence and appearance of LogCFTs in many physical systems are known for a long time, very little has been explored particularly in higher dimensions (see e.g. [30]). In higher dimensional CFTs conformal bootstrap has been used very successfully to study the spectrum, chaos, etc. The idea of conformal bootstrap is to constrain a theory by exploiting the underlying conformal symmetry. The four point function of a CFT can be studied by imposing crossing symmetry and this leads to non-trivial constraint on the spectrum of the theory. The study of conformal bootstrap was initiated in [31-34] which is revived in its modern incarnation in [35] and subsequently studied in [36-45]. For numerical bootstrap unitarity (i.e. positivity of OPE coefficients) plays an important role. Thus within known numerical techniques it's not easy to implement conformal bootstrap to LogCFTs. On the other hand there are analytical methods namely large spin bootstrap [46-59] and Polyakov-Mellin bootstrap [60-64] where one doesn't necessarily require unitarity. This note is our first step toward exploring the plethora of interesting LogCFTs which appear in different branches of physics as mentioned above, using analytic bootstrap. 
We will also see that the constraints imposed by crossing symmetry on the four point correlator dictate the spectrum of the large spin sector of LogCFT. This is an universal sector and any particular LogCFT should contain this at leading order in large spin. In this sense our computation can be considered as a natural starting point to explore any LogCFT. In this article we use the conformal bootstrap techniques in the large spin limit to compute the anomalous dimension of logarithmic operators for a particular type of LogCFT. We also compute it (strictly speaking, for a special case) from effective dual gravitational description where anomalous dimension of the double-trace operators is identified with the binding energy of two rapidly rotating particles inside AdS [48]. By doing so we show that cluster decomposition principle holds true even for non-unitary LogCFTs as long as the scaling dimensions are positive.

The paper is organised as follows. In section 2 we introduce the concept of Logarithmic Generalised Free Field (LGFF) theory and perform the meanfield analysis for rank-2 logarithmic scalar correlators. Section 3 describes how to compute the leading correction to the anomalous dimension of rank-3 even spin double trace logarithmic operators appearing in the spectrum. In section 4 we independently compute the anomalous dimension from a dual bulk effective theory. We conclude with some forward-looking remarks about the possible future directions in section 5. Appendices contain some details of the computation.

\section{Logarithmic generalized free fields}

In this section we consider a particular four dimensional LogCFT (following [30] and the references therein): the logarithmic counterpart of the well-known generalized free fields (GFF). In particular, this is a theory of a rank-two scalar multiplet $\phi_{i}(i=1,2)$ of dimension $\Delta_{\phi}$ with the following two-point function,

$$
\left\langle\phi_{i}(x) \phi_{j}(0)\right\rangle=\frac{1}{|x|^{2 \Delta_{\phi}}}\left(\begin{array}{cc}
-\ln x^{2} & 1 \\
1 & 0
\end{array}\right)_{i j} .
$$

The operator content of the $\phi_{i} \times \phi_{j}$ OPE consists of the unit operator $\mathbb{1}$ as well as a tower of "double-trace" primaries $\mathcal{O}_{i j}^{(n, \ell)}$. For even $\ell$ and fixed $n$ there are three different double-trace operators, whereas for odd $\ell$ there is only one which form a rank- 3 and rank- 1 multiplet respectively. For the simplest case with $n=0$ scalar this logarithmic triplet is defined by the following operators,

$$
S_{1}=\frac{1}{2}:\left(\phi_{1}\right)^{2}:, \quad S_{2}=: \phi_{1} \phi_{2}:, \quad S_{3}=:\left(\phi_{2}\right)^{2}:
$$

where : : denotes normal ordering. Now we would like to bootstrap the four point correlator of four identical scalar rank-2 logarithmic operators.

Let us begin with a brief review of how the bootstrap equation can be used to reproduce the results from the mean field theory correlator in the usual (or non logarithmic) CFT. Consider a four-point correlator of four identical scalar operators $\phi$ of dimension $\Delta_{\phi}$. This 
correlator has a conformal block decomposition is $s$ and $t$ channel.

$$
\begin{aligned}
\left\langle\phi\left(x_{1}\right) \phi\left(x_{2}\right) \phi\left(x_{3}\right) \phi\left(x_{4}\right)\right\rangle & =\frac{1}{\left(x_{12} x_{34}\right)^{2 \Delta_{\phi}}}\left(1+\left(\frac{u}{v}\right)^{\Delta_{\phi}}+u^{\Delta_{\phi}}\right) \\
& =\frac{1}{\left(x_{12} x_{34}\right)^{2 \Delta_{\phi}}} \sum_{\Delta, \ell} c_{\Delta, \ell} \mathcal{G}_{\Delta, \ell}(u, v) \\
& =\frac{1}{\left(x_{13} x_{24}\right)^{2 \Delta_{\phi}}} \sum_{\Delta, \ell} c_{\Delta, \ell} \mathcal{G}_{\Delta, \ell}(v, u),
\end{aligned}
$$

where $x_{i j}=x_{i}-x_{j}$ and the conformal cross ratios are defined as,

$$
u=\frac{x_{12}^{2} x_{34}^{2}}{x_{13}^{2} x_{24}^{2}}=z \bar{z}, \quad v=\frac{x_{14}^{2} x_{23}^{2}}{x_{13}^{2} x_{24}^{2}}=(1-z)(1-\bar{z}) .
$$

We will interchangeably use $u, v$ or $z, \bar{z}$. Here $\Delta, \ell, c_{\Delta, \ell}$, are the dimension, spin and OPE coefficients of the operators getting exchanged in the intermediate channel and $\mathcal{G}_{\Delta, \ell}(u, v)$ are the conformal blocks,

$$
\mathcal{G}_{\Delta, \ell}(u, v)=\frac{1}{2^{\ell}} \frac{z \bar{z}}{z-\bar{z}}\left(k_{2 \ell+\tau}(z) k_{\tau-2}(\bar{z})-k_{2 \ell+\tau}(\bar{z}) k_{\tau-2}(z)\right),
$$

with,

$$
k_{\beta}(x)=x_{2 / 2}^{\beta} F_{1}\left(\frac{\beta}{2}, \frac{\beta}{2} ; \beta ; x\right) .
$$

The equality of the first and the third line of (2.3) reads,

$$
1+v^{-\Delta_{\phi}}+u^{-\Delta_{\phi}}=v^{-\Delta_{\phi}} \sum_{\Delta, \ell} c_{\Delta, \ell} \mathcal{G}_{\Delta, \ell}(v, u) .
$$

In mean field theory the intermediate operators consist of the identity and the double-trace operators $\mathcal{O}_{n, \ell}$ having dimension $\Delta=2 \Delta_{\phi}+2 n+\ell$. Now let us focus on (2.7) in the limit $u \sim 0$. The conformal blocks $\mathcal{G}_{\Delta, \ell}(v, u)$ can be schematically expanded around $u \sim 0$ as follows,

$$
v^{-\Delta_{\phi}} \mathcal{G}_{2 \Delta_{\phi}+2 n, \ell}(v, u) \sim \sum_{m=0}^{\infty} u^{m} f_{m}(v)+\log u \sum_{m=0}^{\infty} u^{m} \tilde{f}_{m}(v) .
$$

Note that the l.h.s. of (2.7) has a power law singularity as $u^{-\Delta_{\phi}}$ whereas each term on the r.h.s. has a $\log u$ singularity. Hence the power law singularity can not be reproduced by summing over a finite number of terms on the r.h.s. . It can be shown [46, 47] that by summing over large spin $\ell \gg 1$ operators in the r.h.s. we can reproduce the l.h.s. of (2.7). This indicates why the large spin operators are important to reproduce the mean field theory of an ordinary CFT.

Now we will discuss the importance of large spin operators in the context of LogCFT following the same steps as above. We consider the four-point function of a rank two scalar 
operator $\phi_{i}$ of dimension $\Delta_{\phi}{ }^{1}$. It was shown in [30] that the four point function can be written as,

$$
\left\langle\phi_{i}\left(x_{1}\right) \phi_{j}\left(x_{2}\right) \phi_{k}\left(x_{3}\right) \phi_{\ell}\left(x_{4}\right)\right\rangle=F_{i j k \ell}\left(u, v, \zeta_{m}\right) P_{\Delta_{\phi} \Delta_{\phi} \Delta_{\phi} \Delta_{\phi}}\left(x_{n}\right)
$$

where,

$$
P_{\Delta_{1} \Delta_{2} \Delta_{3} \Delta_{4}}\left(x_{n}\right)=\prod_{n<r} \frac{1}{\left|x_{n r}\right|^{\Delta_{n}-\Delta_{r}-\Sigma / 3}}, \quad \Sigma:=\sum_{i=1}^{4} \Delta_{i}
$$

and,

$$
\zeta_{m}=\partial_{\Delta_{m}} \ln P_{\Delta_{1} \Delta_{2} \Delta_{3} \Delta_{4}}\left(x_{n}\right) .
$$

$F_{i j k \ell}$ is a tensor which must satisfy the cyclic permutation symmetry under the exchange of $\left(x_{1}, x_{2}, x_{3}, x_{4}\right) \rightarrow\left(x_{2}, x_{3}, x_{4}, x_{1}\right)$ combined by the exchange $x_{1} \leftrightarrow x_{2}$,

$$
F_{i j k \ell}\left(u, v, \zeta_{1}, \zeta_{2}, \zeta_{3}, \zeta_{4}\right)=F_{j k \ell i}\left(v, u, \zeta_{2}, \zeta_{3}, \zeta_{4}, \zeta_{1}\right)=F_{j i k \ell}\left(u / v, 1 / v, \zeta_{2}, \zeta_{1}, \zeta_{3}, \zeta_{4}\right) .
$$

Following the steps mentioned in [30] it can be shown that the constraints imposed by conformal invariance on $F_{i j k \ell}$ allows this to be written in terms of five conformally invariant functions $\mathcal{F}_{\alpha}(u, v), \alpha=1, \cdots 5$ as follows,

$$
\begin{aligned}
F_{1111}= & \mathcal{F}_{1}(u, v)+\sum_{i} \zeta_{i} \mathcal{F}_{2}(u, v)+\left(\zeta_{1} \zeta_{2}+\zeta_{3} \zeta_{4}\right) \mathcal{F}_{3}(u, v) \\
& +\left(\zeta_{1} \zeta_{3}+\zeta_{2} \zeta_{4}\right) \mathcal{F}_{3}(1 / u, v / u)+\left(\zeta_{1} \zeta_{4}+\zeta_{2} \zeta_{3}\right) \mathcal{F}_{3}(v, u) \\
& +\sum_{i<j<k} \zeta_{i} \zeta_{j} \zeta_{k} \mathcal{F}_{4}(u, v)+\zeta_{1} \zeta_{2} \zeta_{3} \zeta_{4} \mathcal{F}_{5}(u, v) \\
F_{1112}= & \mathcal{F}_{2}(u, v)+\zeta_{1} \mathcal{F}_{3}(v, u)+\zeta_{2} \mathcal{F}_{3}(1 / u, v / u)+\zeta_{3} \mathcal{F}_{3}(u, v) \\
& +\left(\zeta_{1} \zeta_{2}+\zeta_{1} \zeta_{3}+\zeta_{2} \zeta_{3}\right) \mathcal{F}_{4}(u, v)+\zeta_{1} \zeta_{2} \zeta_{3} \mathcal{F}_{5}(u, v) \\
F_{1122}= & \mathcal{F}_{3}(u, v)+\left(\zeta_{1}+\zeta_{2}\right) \mathcal{F}_{4}(u, v)+\zeta_{1} \zeta_{2} \mathcal{F}_{5}(u, v) \\
F_{1222}= & \mathcal{F}_{4}(u, v)+\zeta_{1} \mathcal{F}_{5}(u, v) \\
F_{2222}= & \mathcal{F}_{5}(u, v)
\end{aligned}
$$

Hence the conformal bootstrap constraint on the correlator essentially reduces to the following crossing symmetry condition on the functions $\mathcal{F}_{\alpha}(u, v)$,

$$
\begin{aligned}
\mathcal{F}_{\alpha}(u, v) & =\mathcal{F}_{\alpha}(v, u)=\mathcal{F}_{\alpha}(u / v, 1 / v) \quad \text { for } \quad \alpha=1,2,4,5 ; \\
\mathcal{F}_{3}(u, v) & =\mathcal{F}_{3}(u / v, 1 / v) .
\end{aligned}
$$

Each of these functions can be decomposed into conformal blocks which results in five bootstrap equations for $\mathcal{F}_{\alpha}(u, v)$. Let us focus on the bootstrap equation for $\mathcal{F}_{2}(u, v)$,

$$
\mathcal{F}_{2}(u, v)=\mathcal{F}_{2}(v, u)
$$

\footnotetext{
${ }^{1}$ For simplicity we consider only rank two operators. However, this can be generalised to higher rank operators as well.
} 
In this case the exchange operators in each channel are rank- $r$ operator $(r=3$ for even spin and $r=1$ for odd spin) $\mathcal{O}_{p}$ of spin $\ell$ having the three-point function $\left\langle\phi_{i} \phi_{j} \mathcal{O}_{p}\right\rangle$ characterized by the following OPE coefficients $\lambda_{i j p}$,

$$
\begin{array}{llll}
\lambda_{11 p}=a_{p}, & \lambda_{12 p}=\lambda_{21 p}=b_{p} \lambda_{22 p}=c_{p} & \text { for even } \ell, & p=1,2,3, \\
\lambda_{11 p}=0, & \lambda_{12 p}=-\lambda_{21 p}=\tilde{b}_{p}, \quad \lambda_{22 p}=0, & \text { for odd } \ell, & p=1 .
\end{array}
$$

The conformal block decomposition for $\mathcal{F}_{2}(u, v)$ is given by summing over even and odd spin rank- $r$ operators $\mathcal{O}_{p}$ with dimension $\Delta$ and spin $\ell$,

$$
\mathcal{F}_{2}(u, v)=\sum_{\mathcal{O}} \mathcal{D}_{\mathcal{O}} G_{\Delta, \ell}\left(u, v, \Delta_{i}\right)
$$

where,

$$
\begin{aligned}
\mathcal{D}_{\mathcal{O}} & =\sum_{p, q=1}^{r}\left[a_{p}+b_{p}\left(\partial_{\Delta_{1}}+\partial_{\Delta_{2}}\right)+c_{p} \partial_{\Delta_{1}} \partial_{\Delta_{2}}\right]\left[b_{q}+c_{q} \partial_{\Delta_{3}}\right] V^{p q}\left(\partial_{\mathcal{O}}\right) \quad \text { for even } \ell \\
\mathcal{D}_{\mathcal{O}} & =\sum_{p, q=1}^{r} \tilde{b}_{p} \tilde{b}_{q}\left(\partial_{\Delta_{2}}-\partial_{\Delta_{1}}\right) V^{p q}\left(\partial_{\mathcal{O}}\right) \quad \text { for odd } \ell \\
V^{p q}(\partial) & = \begin{cases}\partial^{n} / n !, & \text { if } n=p+q-r-1 \geq 0 \\
0, & \text { if } n<0\end{cases}
\end{aligned}
$$

and the $G_{\Delta, \ell}$ 's are defined in (2.24). For identical scalars the odd spin contribution vanishes because of the following identity (see [65-67]),

$$
\left.\frac{\partial}{\partial_{\Delta_{1}}} G_{\Delta, \ell}\left(u, v, \Delta_{i}\right)\right|_{\Delta_{i}=\Delta_{\phi}}=\cdots=\left.\frac{\partial}{\partial_{\Delta_{4}}} G_{\Delta, \ell}\left(u, v, \Delta_{i}\right)\right|_{\Delta_{i}=\Delta_{\phi}}=\left.\frac{1}{12} \log \left(\frac{v}{u^{2}}\right) G_{\Delta, \ell}\left(u, v, \Delta_{i}\right)\right|_{\Delta_{i}=\Delta_{\phi}} .
$$

The four point function in the mean field theory can be computed using Wick's theorem and is given by [30],

$$
\mathcal{F}_{2}(v, u)=\frac{1}{6}\left[\left(\frac{v}{u^{2}}\right)^{\Delta_{\phi} / 3} \log \left(\frac{v}{u^{2}}\right)+\left(\frac{u}{v^{2}}\right)^{\Delta_{\phi} / 3} \log \left(\frac{u}{v^{2}}\right)+(u v)^{\Delta_{\phi} / 3} \log (u v)\right] .
$$

In this case the operators $\mathcal{O}_{n, \ell}$ have known OPE coefficient $q_{n, \ell}[68]$,

$$
q_{n, \ell}=\frac{2^{\ell}\left(\Delta_{1}+1-h\right)_{n}\left(\Delta_{2}+1-h\right)_{n}\left(\Delta_{1}\right)_{\ell+n}\left(\Delta_{2}\right)_{\ell+n}}{\ell ! n !(h+\ell)_{n}\left(\Delta_{1}+\Delta_{2}+n+1-2 h\right)_{n}\left(\ell+2 n+\Delta_{1}+\Delta_{2}-1\right)_{\ell}\left(\Delta_{1}+\Delta_{2}+\ell+n-h\right)_{n}}
$$

where $h=d / 2=2,(a)_{b}=\frac{\Gamma(a+b)}{\Gamma(b)}$. The $s$-channel decomposition of the correlator is given by,

$$
\mathcal{F}_{2}(u, v)=\frac{1}{6}\left(\frac{v}{u^{2}}\right)^{\Delta_{\phi} / 3} \log \left(\frac{v}{u^{2}}\right)+\left.\sum_{\ell, n=0}^{\infty} \mathcal{D}^{(n, \ell)} G_{\Delta, \ell}\left(u, v, \Delta_{i}\right)\right|_{\Delta_{i}=\Delta_{\phi}, \Delta=2 \Delta_{\phi}+2 n+\ell}
$$


with,

$$
\begin{aligned}
\mathcal{D}^{(n, \ell)} & =\partial_{\Delta_{\phi}} q_{n, \ell}+q_{n, \ell}\left(2 \partial_{\Delta}+\partial_{\Delta_{1}}+\partial_{\Delta_{2}}+2 \partial_{\Delta_{3}}\right) \\
G_{\Delta, \ell}\left(u, v, \Delta_{i}\right) & =u^{-\frac{1}{6}\left(\Delta_{1}+\Delta_{2}+\Delta_{3}+\Delta_{4}\right)} v^{\frac{1}{6}\left(-\Delta_{1}+2 \Delta_{2}+2 \Delta_{3}-\Delta_{4}\right)} \mathcal{G}_{\Delta, \ell}(u, v) .
\end{aligned}
$$

For simplicity we will focus on the double trace operators $\mathcal{O}_{0, \ell}$ with $n=0$. The bootstrap equation (2.15) is in general quite complicated to solve and we will see that it simplifies in the limit $v \ll u \ll 1$ and $\ell \gg 1$. In this limit we approximate $\bar{z} \sim 1-v, z \sim u$ and the leading term in (2.20) is given by,

$$
\mathcal{F}_{2}(v, u) \sim \frac{1}{6}\left(\frac{u}{v^{2}}\right)^{\Delta_{\phi} / 3} \log \left(\frac{u}{v^{2}}\right) .
$$

In (2.22) the first term is the contribution from the identity operator exchange. We will now use (2.15) to reproduce (2.25) from the $s$ - channel of (2.15). In the $\ell \gg 1$ limit the OPE coefficient and its derivative can be approximated as follows,

$$
\begin{aligned}
q_{0, \ell} & \sim \frac{\sqrt{\pi} 2^{-2 \Delta_{\phi}-\ell+2} \ell^{2 \Delta_{\phi}-\frac{3}{2}}}{\Gamma^{2}\left(\Delta_{\phi}\right)}, \\
\partial_{\Delta_{\phi}} q_{0, \ell} & \sim-\frac{\sqrt{\pi} 2^{-2 \Delta_{\phi}-\ell+3} \ell^{2 \Delta_{\phi}-\frac{3}{2}}}{\Gamma^{2}\left(\Delta_{\phi}\right)}\left(\psi\left(\Delta_{\phi}\right)-\log \ell+\log 2\right),
\end{aligned}
$$

where $\psi$ is the digamma function. For large $\ell$ and $n=0$ we can approximate (2.22) by,

$$
\begin{aligned}
\mathcal{F}_{2}(u, v) \sim & \beta \sum_{\ell \gg 1}-\frac{4^{2} \ell^{2 \Delta_{\phi}-1} u^{\Delta_{\phi}}\left(\psi\left(\Delta_{\phi}\right)+\log (2)-\log \ell\right)}{\Gamma^{2}\left(\Delta_{\phi}\right)} K_{0}(2 \ell \sqrt{v}) \\
& +\beta \log u \sum_{\ell \gg 1} \frac{2^{3-2 \Delta_{\phi}} \ell^{2 \Delta_{\phi}-1} u^{\Delta_{\phi}}}{\Gamma^{2}\left(\Delta_{\phi}\right)} K_{0}(2 \ell \sqrt{v}) \\
& +\beta \frac{1}{3} \log \left(\frac{v}{u^{2}}\right) \sum_{\ell \gg 1} \frac{2^{3-2 \Delta_{\phi}} \ell^{2 \Delta_{\phi}-1} u^{\Delta_{\phi}}}{\Gamma^{2}\left(\Delta_{\phi}\right)} K_{0}(2 \ell \sqrt{v}),
\end{aligned}
$$

where,

$$
\beta=u^{-2 \Delta_{\phi} / 3} v^{\Delta_{\phi} / 3},
$$

and $K_{0}$ is a modified Bessel function of the second kind. We can approximate the large $\ell$ sum by an integral $\sum_{\ell \gg 1} \rightarrow \frac{1}{2} \int d \ell$ where the $\frac{1}{2}$ indicates that we are summing over only even spin operators. The integrals we need are of the following kind (see $[69,70]$ for similar analysis),

$$
\begin{aligned}
\int_{\ell_{0}}^{\infty} \ell^{2 \Delta_{\phi}-1-a} K_{0}(2 \ell \sqrt{v}) \log \ell & =-\frac{v^{-\Delta_{\phi}+a / 2} \Gamma^{2}\left(\Delta_{\phi}-a / 2\right)}{8}\left(\log v-2 \psi\left(\Delta_{\phi}-a / 2\right)\right)+\cdots, \\
\int_{\ell_{0}}^{\infty} \ell^{2 \Delta_{\phi}-1-a} K_{0}(2 \ell \sqrt{v}) & =\frac{1}{4} v^{-\frac{a}{2}-\Delta_{\phi}} \Gamma^{2}\left(\frac{a}{2}+\Delta_{\phi}\right)+\cdots
\end{aligned}
$$


where the dots denote the subleading terms in $v$. Using this in (2.28) we get,

$$
\mathcal{F}_{2}(u, v) \sim \frac{1}{6}\left(\frac{u}{v^{2}}\right)^{\Delta_{\phi} / 3} \log \left(\frac{u}{v^{2}}\right) .
$$

Thus we see that the leading behavior of $t$-channel in the limit $v \ll u \ll 1$ is reproduced by summing over large spin double trace operators in the $s$-channel of $(2.15)$.

The above result may seem like a mere consistency check. But it is worth noting that, just like GFF, given a primary operator $\mathcal{O}$ with twist $\tau$ in a LogCFT at large spin there must exist an infinite tower of primaries with twist $2 \tau+n$ where $n=0,1,2, \ldots$ in order to satisfy crossing symmetry. Since this is just consequence of crossing symmetry this large spin spectrum is universal i.e. independent of particular LogCFT. In this sense, the starting point of large spin analysis for any given LogCFT should be identical to this section.

\section{Computing the anomalous dimensions at large $\ell$}

In this section we will use the bootstrap equation (2.15) to compute the anomalous dimension of the large spin operators $\mathcal{O}_{0, \ell}$ in an interacting LogCFT with the following dimension,

$$
\Delta=2 \Delta_{\phi}+\ell+\gamma_{0, \ell} .
$$

In order to do so we focus on the subleading corrections to (2.15) in the $v \ll u \ll 1$ limit. To compute the anomalous dimension we need to focus on the coefficient of $\gamma_{0, \ell} u^{\Delta_{\phi}} \log u$ from (2.22).

$$
\left.\left.\mathcal{F}_{2}(u, v)\right|_{\log u} \sim \sum_{\ell \gg 1} \mathcal{D}^{(n, \ell)} G\left(u, v, \Delta, \Delta_{i}\right)\right|_{\log u} .
$$

At this point we assume that $\gamma_{0, \ell}$ has the following expansion in the $\ell \gg 1$ limit,

$$
\gamma_{0, \ell} \sim \frac{\gamma_{0}}{\ell^{a}}+\cdots,
$$

where the dots denote the subleading terms in $\ell$. We have to determine the constant $a$ and the $\ell$ independent piece $\gamma_{0}$ from the bootstrap equation. To extract the $\log u$ term above, we have to use the integrals (see (2.30)). This results in the following term from (3.2),

$$
\left.\mathcal{F}_{2}(u, v)\right|_{\log u} \sim \gamma_{0} u^{\Delta_{\phi} / 3} v^{\left(3 a-4 \Delta_{\phi}\right) / 6} \frac{\Gamma^{2}\left(\Delta_{\phi}-\frac{a}{2}\right)}{\Gamma^{2}\left(\Delta_{\phi}\right)}\left(\psi\left(\Delta_{\phi}-\frac{a}{2}\right)-\psi\left(\Delta_{\phi}\right)\right) .
$$

Now we focus on the $t$-channel. The $t$-channel has an expansion controlled by the twist of the exchange operator and the subleading correction comes from the minimal twist operators $\mathcal{O}_{m}$ of spin $\ell_{m}$, dimension $\Delta_{m}$ and twist $\tau_{m}$. We assume that the operator dimensions are always positive and $\tau_{m}>0$. We will focus on the coefficient of $\log u$ which comes from the $t$ channel and match it with (3.4). We will use the following expansion for the $t$-channel conformal block [46],

$$
\mathcal{G}_{\tau_{m}, \ell_{m}}(v, u) \sim C_{m} v^{\frac{\tau_{m}}{2}}(1-u)^{\ell_{m}}{ }_{2} F_{1}\left(\frac{\tau_{m}}{2}+\ell_{m}, \frac{\tau_{m}}{2}+\ell_{m}, \tau_{m}+2 \ell_{m}, 1-u\right) .
$$


At small $u$ we have,

${ }_{2} F_{1}(\beta, \beta, 2 \beta ; 1-u)=\frac{\Gamma(2 \beta)}{\Gamma^{2}(\beta)} \sum_{n=0}^{\infty}\left(\frac{(\beta)_{n}}{n !}\right)^{n} u^{n}\left[2\left(\psi(n+1)-\psi\left(\frac{\tau_{m}}{2}+\ell_{m}+n\right)\right)-\log u\right]$.

To obtain the $\log u$ term we will also need the expression for derivative of $\mathcal{G}(v, u)$ w.r.t. $\Delta$ which is given by,

$$
\begin{aligned}
\partial_{\Delta} \mathcal{G}_{\Delta, \ell}(v, u)=\frac{1}{2^{\ell}} \frac{(1-z)(1-\bar{z})}{\bar{z}-z}( & \partial_{\Delta} k_{2 \ell+\tau}(1-\bar{z}) k_{\tau-2}(1-z)+k_{2 \ell+\tau}(1-\bar{z}) \partial_{\Delta} k_{\tau-2}(1-z) \\
& \left.-\partial_{\Delta} k_{2 \ell+\tau}(1-z) k_{\tau-2}(1-\bar{z})-k_{2 \ell+\tau}(1-z) \partial_{\Delta} k_{\tau-2}(1-\bar{z})\right) .
\end{aligned}
$$

A typical term in the above expression gives,

$$
\partial_{\Delta} k_{\Delta+\ell}(x)=\frac{1}{2} \log x k_{\Delta+\ell}(x)+x^{\frac{\Delta+\ell}{2}} \partial_{\Delta}{ }_{2} F_{1}\left(\frac{\Delta+\ell}{2}, \frac{\Delta+\ell}{2}, \Delta+\ell, x\right) .
$$

In the small $u$ limit, the $\log u$ terms can appear from the derivative of hypergeometric pieces as follows,

$$
\partial_{\Delta} \mathcal{G}_{\Delta, \ell}(v, u) \sim \frac{1}{2^{\ell}} v^{\frac{\tau}{2}}(1-u)^{\frac{\tau}{2}+\ell} \partial_{\Delta}{ }_{2} F_{1}\left(\frac{\Delta+\ell}{2}, \frac{\Delta+\ell}{2}, \Delta+\ell, 1-u\right) .
$$

Similarly for $r^{\text {th }}$ derivative $\log u$ dependent terms can arise from,

$$
\partial_{\Delta}^{r} \mathcal{G}_{\Delta, \ell}(v, u) \sim \frac{1}{2^{\ell}} v^{\frac{\tau}{2}}(1-u)^{\frac{\tau}{2}+\ell} \partial_{\Delta}^{r}{ }_{2} F_{1}\left(\frac{\Delta+\ell}{2}, \frac{\Delta+\ell}{2}, \Delta+\ell, 1-u\right) .
$$

To collect the contribution to the coefficients of $\log u$ we need to use the integral representation of hypergeometric function, [71]),

$$
{ }_{2} F_{1}\left(\frac{\Delta+\ell}{2}, \frac{\Delta+\ell}{2}, \Delta+\ell, 1-u\right)=\frac{\Gamma(\Delta+\ell)}{2 \pi i \Gamma^{4}\left(\frac{\Delta+\ell}{2}\right)} \int_{-i \infty}^{i \infty} \Gamma^{2}\left(\frac{\Delta+\ell}{2}+t\right) \Gamma^{2}(-t) u^{t} d t .
$$

Since $u \ll 1$ we close the contour to the right hand side and pick residue from the double pole from $\Gamma^{2}(-t)$. It will produce two kind of terms: $u^{0}$ (non-log term) and $u^{0} \log u(\log u$ term). The relevant terms that can give $\log u$ terms in the $t$-channel are given by,

$$
\begin{aligned}
& \left.\mathcal{D}_{\mathcal{O}} G(v, u)\right|_{\text {relevant }} \\
& =\sum_{p, q=1}^{r} a_{p} b_{q} V^{p q} G(v, u)+\left(a_{p} c_{q}+2 b_{p} b_{q}\right) \partial_{1} V^{p q} G(v, u) \\
& =\sum_{p, q=1}^{r} a_{p} b_{q}\left(\frac{u}{v^{2}}\right)^{\Delta_{\phi} / 3}\left[\frac{\partial^{m}}{m !} \mathcal{G}_{\Delta, \ell}(v, u)\right]_{u^{0} \log u} \\
& \quad+\sum_{p, q=1}^{r}\left(a_{p} c_{q}+2 b_{p} b_{q}\right) \frac{1}{12}(\log u)\left(\frac{u}{v^{2}}\right)^{\Delta_{\phi} / 3}\left[\frac{\partial^{m}}{m !} \mathcal{G}_{\Delta, \ell}(v, u)\right]_{u^{0}},
\end{aligned}
$$


where $m=p+q-r-1$ from (2.18). Notice that all other terms will have $(\log u)^{2},(\log u)(\log v)$ etc. Here we are interested in only $\log u$ terms. For minimal twist operator exchange in t-channel,

$$
\partial_{\Delta}^{m} \mathcal{G}_{\tau_{m}, \ell_{m}}(v, u) \sim \frac{1}{2_{m}^{\ell}} v^{\frac{\tau_{m}}{2}}\left[I_{\log u}^{m}\left(\tau_{m}, \ell_{m}\right)+I_{n o n-\log u}^{m}\left(\tau_{m}, \ell_{m}\right)\right]
$$

where,

$$
\begin{aligned}
I_{\log u}^{m}(\tau, \ell)= & \left.\partial_{\Delta}^{m}\left(\frac{\Gamma(\Delta+\ell)}{\Gamma^{4}\left(\frac{\Delta+\ell}{2}\right)} \Gamma^{2}\left(\frac{\Delta+\ell}{2}+t\right)\right)\right|_{t=0, \Delta=\tau+\ell}, \\
I_{n o n-\log u}^{m}(\tau, \ell)= & \left.2 \gamma_{E} \partial_{\Delta}^{m}\left(\frac{\Gamma(\Delta+\ell)}{\Gamma^{4}\left(\frac{\Delta+\ell}{2}\right)} \Gamma^{2}\left(\frac{\Delta+\ell}{2}+t\right)\right)\right|_{t=0, \Delta=\tau+\ell} \\
& +\left.\partial_{\Delta}^{m} \partial_{t}\left(\frac{\Gamma(\Delta+\ell)}{\Gamma^{4}\left(\frac{\Delta+\ell}{2}\right)} \Gamma^{2}\left(\frac{\Delta+\ell}{2}+t\right)\right)\right|_{t=0, \Delta=\tau+\ell}
\end{aligned}
$$

Finally, the coefficient of $\log u$ term in the t-channel is given by,

$$
\begin{aligned}
\left.\mathcal{F}_{2}(v, u)\right|_{\log u}= & \left.\mathcal{D}_{\mathcal{O}} G(v, u)\right|_{\log u} \\
= & \sum_{p, q=1}^{r} a_{p} b_{q}\left(\frac{u}{v^{2}}\right)^{\Delta_{\phi} / 3}\left[\frac{1}{m ! 2^{\ell_{m}}} v^{\tau_{m} / 2} I_{\log u}^{m}\left(\tau_{m}, \ell_{m}\right)\right] \\
& +\sum_{p, q=1}^{r}\left(a_{p} c_{q}+2 b_{p} b_{q}\right) \frac{1}{12}\left(\frac{u}{v^{2}}\right)^{\Delta_{\phi} / 3}\left[\frac{1}{m ! 2^{\ell_{m}}} v^{\tau_{m} / 2} I_{n o n-\log u}^{m}\left(\tau_{m}, \ell_{m}\right)\right] .
\end{aligned}
$$

Comparing (3.4) and (3.15) we can see that the bootstrap equation is satisfied if $a=\tau_{m}$. Clearly, this expansion (3.3) is valid only when $\tau_{m}>0$ which may not be true for any nonunitary theory where there is a possibility that $\Delta_{m}<0$. Hence this is true only for a subsector of nonunitary theories where the dimensions are always positive. This results in the following anomalous dimension,

$$
\begin{aligned}
\gamma_{0}= & \frac{2 \Gamma^{2}\left(\Delta_{\phi}\right)}{\Gamma^{2}\left(\Delta_{\phi}-\frac{\tau_{m}}{2}\right)\left(-\psi\left(\Delta_{\phi}\right)+\psi\left(\Delta_{\phi}-\frac{\tau_{m}}{2}\right)\right)} \\
& \times \sum_{p, q=1}^{r} \frac{1}{m ! 2^{\ell_{m}}}\left[a_{p} b_{q} I_{\log u}^{m}\left(\tau_{m}, \ell_{m}\right)+\frac{1}{12}\left(a_{p} c_{q}+2 b_{p} b_{q}\right) I_{n o n-\log u}^{m}\left(\tau_{m}, \ell_{m}\right)\right] .
\end{aligned}
$$

As long as there is no operator with negative scaling dimension in the theory, the anomalous dimension $\gamma_{0, \ell} \sim \frac{\gamma_{0}}{\ell^{\tau} m}$ with $\tau_{m}>0$ and in the strict $\ell \rightarrow \infty$ limit $\gamma_{0, \ell}$ vanishes. This suggests that cluster decomposition holds even for LogCFTs with positive scaling dimensions, which are necessarily non-unitary. In the next section we will see this has nice bulk interpretation as well. It means that two corresponding particles which are rapidly rotating in AdS space are well separated from each other.

\section{The dual gravity picture}

The expression for the anomalous dimension derived above in (3.16) is the main result of this note. Since there exists a holographic model dual to LogCFTs, it would be nice to 
see if the anomalous dimension can be interpreted (at least the parametric behaviour) via holography. There are some works in the literature that study dual of LogCFTs [24, 25, 72]. Here we consider a particular case of the main result, namely we consider a simplified setup where the minimal twist, $\tau_{m}=2$. Consequently we can have a simple ${ }^{2}$ dual gravity description in the bulk. In this setup we try to compute the anomalous dimension of the exchanged operator for LogCFT derived above from dual classical gravity. We follow the formalism in [48] to obtain the anomalous dimension. It is known from the literature [24, 25, 72], dual gravitational effective theory of a LogCFT is given by some higher derivative EOM. E.g. for a rank-r LogCFT the dual scalar field in AdS satisfies the following EOM,

$$
\left(\square-M^{2}\right)^{r} \Phi_{\mathrm{LCFT}}^{(r)}=0 .
$$

In this paper we focus on rank-2 multiplet and therefore the EOM

$$
\begin{array}{r}
\left(\square-M^{2}\right)^{2} \Phi_{\mathrm{LCFT}}=0 \\
\left(\square-M^{2}\right) \underbrace{\left(\square-M^{2}\right) \Phi_{\mathrm{LCFT}}}_{\Phi_{\mathrm{CFT}}}=0 .
\end{array}
$$

By definition $\Phi_{\mathrm{CFT}}$ is the solution of KG equation in $\mathrm{AdS}_{d+1},{ }^{3}$

$$
d s^{2}=\frac{1}{\cos ^{2} \rho}\left(-d t^{2}+d \rho^{2}+\sin ^{2} \rho d \Omega_{d-1}^{2}\right)
$$

where $\rho=\frac{\pi}{2}$ is the boundary and we have taken the AdS radius to be one. We will be using global metric because the connection between fields in AdS and operators with definite scaling dimension in the CFT is more transparent in global coordinates than in Poincare patch. The solution to the EOM is known $[73,74]$ and is given by,

$$
\Phi_{\mathrm{CFT}}(x)=\sum_{n, \ell, J} \phi_{n \ell J}(x) a_{n \ell J}+\phi_{n \ell J}^{*}(x) a_{n \ell J}^{\dagger},
$$

where,

$$
\begin{aligned}
\phi_{n \ell J} & =\frac{1}{N_{\Delta, n, \ell}} e^{i \omega_{n, \ell} t} Y_{\ell J}(\Omega) \sin ^{\ell} \rho \cos ^{\Delta} \rho_{2} F_{1}\left(-n, \Delta+n+\ell, \ell+\frac{d}{2}, \sin ^{2} \rho\right) \\
\omega_{n, \ell} & \equiv \Delta+2 n+\ell, \quad M^{2} \equiv \Delta(\Delta-d) \\
N_{\Delta, n, \ell} & \equiv(-1)^{n} \sqrt{\frac{n ! \Gamma^{2}\left(\ell+\frac{d}{2}\right) \Gamma\left(\Delta+n-\frac{d-2}{2}\right)}{\Gamma\left(n+\ell+\frac{d}{2}\right) \Gamma(\Delta+n+\ell)}}
\end{aligned}
$$

and $a_{n \ell J}^{\dagger}, a_{n \ell J}$ are creation and annihilation operators respectively.

\footnotetext{
${ }^{2}$ Of course this won't reproduce the full parametric behaviour of the anomalous dimension. We leave that detailed computation for future work.

${ }^{3}$ The bulk analysis is valid for arbitrary $d$. However in order to compare this with the LogCFT we will explicitly choose $d=4$.
} 
Solution to the bulk EOM. To obtain $\Phi_{\mathrm{LCFT}}$ (which we call $\Phi$ now onward for brevity) one needs to solve the following differential equation

$$
\left(\square-M^{2}\right) \Phi=\Phi_{\mathrm{CFT}}
$$

This is nothing but KG equation with a known source term $\Phi_{n, \ell, J}$ which is the solution to the homogeneous $\mathrm{KG}$ equation in $\mathrm{AdS}_{d+1}$. The standard approach to solve such an inhomogeneous partial differential equation is by using Green function method. For that we need to solve the KG equation with a delta function source

$$
\left(\square-M^{2}\right) G\left(x-x^{\prime}\right)=\delta^{d+1}\left(x-x^{\prime}\right),
$$

then integrate that solution over the known function $\Phi_{\mathrm{CFT}}$

$$
\Phi(x)=\int \sqrt{g} d^{d+1} x^{\prime} G\left(x-x^{\prime}\right) \Phi_{\mathrm{CFT}}\left(x^{\prime}\right) .
$$

The bulk-to-bulk propagator (Green functions) can be written as an infinite sum over the normalizable modes using the general Green's function formula [73]

$$
i G\left(x, x^{\prime}\right)=\int \frac{d \omega}{2 \pi} \sum_{n^{\prime}, \ell^{\prime}, \vec{m}^{\prime}} e^{i \omega\left(t-t^{\prime}\right)} \frac{\phi_{n, \ell, \vec{m}}^{*}(\vec{x}) \phi_{n, \ell, \vec{m}}\left(\vec{x}^{\prime}\right)}{\omega_{n^{\prime}, \ell^{\prime}}^{2}-\omega^{2}},
$$

where the normalizable modes $\phi_{n, \ell, \vec{m}}(x)$ are known functions,

$$
\phi_{n, \ell, \vec{m}}(x)=Y_{\ell m}(\Omega) \sin ^{\ell} \rho \cos ^{\Delta} \rho P_{n}^{\ell+d / 2-1, \nu}(\cos 2 \rho),
$$

where $P_{n}^{m, \nu}(\cos 2 \rho)$ are Jacobi polynomials. From (4.5), (4.10) and (4.11) we can get the solution for (4.8) as follows (see appendix B for details),

$$
\begin{aligned}
\Phi(x)= & \int \sqrt{-g} d^{d+1} x^{\prime} G\left(x, x^{\prime}\right) \Phi_{\mathrm{CFT}}\left(x^{\prime}\right) \\
= & \frac{Y_{\ell J}^{*}(\Omega) e^{i \omega_{n, \ell} t}}{i N_{\Delta, n, \ell}} \frac{n !}{\left(\ell+\frac{d}{2}\right)_{n}}(\sin \rho)^{\ell}(\cos \rho)^{\Delta} \sum_{m=0}^{\infty} \frac{1}{\left(\omega_{m, \ell}^{2}-\omega_{n, \ell}^{2}\right)} P_{m}^{\ell+d / 2-1, \nu}(\cos 2 \rho) \\
& \times \int_{0}^{\pi / 2} d \rho^{\prime}\left(\sin \rho^{\prime}\right)^{2 \alpha+1}\left(\cos \rho^{\prime}\right)^{2 \beta-1} P_{m}^{\alpha, \nu}\left(\cos 2 \rho^{\prime}\right) P_{n}^{\alpha, \nu}\left(\cos 2 \rho^{\prime}\right) \\
\equiv & \frac{Y_{\ell J}^{*}(\Omega) e^{i \omega_{n, \ell} t}}{i N_{\Delta, n, \ell}} \frac{n !}{\left(\ell+\frac{d}{2}\right)_{n}}(\sin \rho)^{\ell}(\cos \rho)^{\Delta} f(\rho),
\end{aligned}
$$

where $\sqrt{-g}=\left(\sin \rho^{\prime}\right)^{-d-1}\left(\cos \rho^{\prime}\right)^{d-1}$, and $\alpha=\ell+\frac{d}{2}-1 ; \quad \beta=\Delta-\frac{d}{2}=\nu$.

To obtain the solution $\Phi(x)$ one needs to compute $f(\rho)$ by performing the $m$ sum and $\rho^{\prime}$ integral. The alternative way would be to use (4.13) as an ansatz to the differential equation (4.8), and solve for $f(\rho)$. Pursuing the latter route we end up with the following differential equation

$$
f^{\prime \prime}(\rho)-((2 \Delta-3) \tan \rho-(2 \ell+3) \cot \rho) f^{\prime}(\rho)-(\Delta-\omega+\ell)(\Delta+\omega+\ell) f(\rho)=\sec ^{2} \rho,
$$


which can be solved exactly (see appendix B for details) to get the solution

$$
f(\rho)=-\gamma_{E} \frac{(-1)^{n} \Gamma(\Delta-2) \Gamma(n+\ell+2)}{\Gamma(n+\Delta-1) \Gamma(\ell+2)}{ }_{2} F_{1}\left(-n, n+\ell+\Delta ; \ell+2 ; \sin ^{2} \rho\right) .
$$

Finally the full solution to (4.8) is given by,

$$
\begin{aligned}
\Phi(x)= & -\gamma_{E} \frac{Y_{\ell J}^{*}(\Omega) e^{i \omega_{n, \ell} t}}{i N_{\Delta, n, \ell}}(\sin \rho)^{\ell}(\cos \rho)^{\Delta} \frac{n !}{\left(\ell+\frac{d}{2}\right)_{n}} \frac{\Gamma(\Delta-2) \Gamma(n+\ell+2)}{\Gamma(n+\Delta-1) \Gamma(\ell+2)} \\
& \times{ }_{2} F_{1}\left(-n, n+\ell+\Delta ; \ell+2 ; \sin ^{2} \rho\right) .
\end{aligned}
$$

The anomalous dimension as binding energy. Since we know the solution to the dual bulk scalar field in AdS, following [46, 48, 74] we can try to extract the anomalous dimension as binding energy of two-particle state. As we have mentioned before, global AdS is very useful to have such a bulk interpretation because time translations in global AdS are generated by the dilatation operator $D$ of the dual CFT, and therefore anomalous dimensions in the CFT are equivalent to energy shifts of bulk states due to interactions.

Here we calculate the first order shift in energy due to (Newtonian) gravitational interaction between the two rapidly orbiting particles. To simplify the computation, following [48], we fix one of them at the center of $\mathrm{AdS}_{5},{ }^{4}$ and the other one will be moving with an effective large orbital angular momentum $\ell_{\text {orb }}$. This is equivalent to studying orbital motion of a massive particle in $\mathrm{AdS}_{5}$-Schwarzschild black hole.

$$
d s^{2}=N(r) d t^{2}-\frac{1}{N(r)} d r^{2}-r^{2} d \Omega_{3}^{2}
$$

with,

$$
N(r)=1-\frac{\mu}{r^{2}}+\frac{r^{2}}{R_{\text {AdS }}^{2}}
$$

and the mass of the $\mathrm{BH}$ is given by, $M_{B H}=\frac{3 \operatorname{vol}\left(S^{3}\right)}{16 \pi G_{N}} \mu$. Note that the coordinate $r$ of this metric is related to (4.3) via the map: $r=\tan \rho$. The wave function in $r$ coordinate reduces to,

$$
\begin{aligned}
\Phi(x)= & \underbrace{\left(\frac{1}{\epsilon}-\gamma_{E}\right) \frac{1}{i N_{\Delta, n, \ell_{\text {orb }}}} \frac{n !}{\left(\ell_{\text {orb }}+\frac{d}{2}\right)_{n}} \frac{\Gamma(\Delta-2) \Gamma\left(-\ell_{\text {orb }}-1\right)}{\Gamma(n+\Delta-1) \Gamma\left(-n-\ell_{\text {orb }}-1\right)}}_{\mathcal{N}} \\
& \times \underbrace{\frac{r^{\ell_{\text {orb }}}}{\left(1+r^{2}\right)^{\frac{\ell_{\text {orb }}}{2}}} \frac{1}{\left(1+r^{2}\right)^{\frac{\Delta}{2}}} 2 F_{1}\left(-n, n+\ell_{\text {orb }}+\Delta ; \ell_{\text {orb }}+2 ; \frac{r^{2}}{1+r^{2}}\right)}_{\mathcal{R}(r)} \\
& \times Y_{\ell J}^{*}(\Omega) e^{i \omega_{n, \ell_{\text {orb }}} t} .
\end{aligned}
$$

\footnotetext{
${ }^{4}$ Notice that the bulk can be of arbitrary spacetime dimensions. We are choosing $\mathrm{AdS}_{5}$ because in the CFT side we have used $4 d$ conformal block expressions. Presumably the whole setup will go through for any $d \geq 3$.
} 
We are interested in computing the energy shift of the orbiting particle due to gravitational attraction. In first oder in perturbation the shift in energy is given by,

$$
\begin{aligned}
\delta E_{\text {orb }} & =\left\langle n, \ell_{\text {orb }}|\delta H| n, \ell_{\text {orb }}\right\rangle \\
& =-\frac{\mu}{4} \int d r r^{3} d^{3} \Omega\left\langle n, \ell_{\text {orb }}\left|\left(\frac{r^{-2}}{\left(1+r^{2}\right)^{2}}\left(\partial_{t} \phi\right)^{2}+r^{-2}\left(\partial_{r} \phi\right)^{2}\right)\right| n, \ell_{\text {orb }}\right\rangle, \\
\delta E_{\text {orb }}\left(n, \ell_{\text {orb }}\right) & =-\frac{\mu}{2}|\mathcal{N}|^{2} \int r d r\left(\frac{1}{\left(1+r^{2}\right)^{2}} \omega_{\Delta n \ell_{\text {orb }}}^{2}|\mathcal{R}(r)|^{2}+\left(\partial_{r} \mathcal{R}(r)\right)^{2}\right),
\end{aligned}
$$

where $\mathcal{R}(r)$ is the radial part of the wavefunction. We want to compute this binding energy and compare that with the anomalous dimension $\gamma_{0}$ given in (3.16). Therefore it will suffice if we focus on the regime: $n=0$ and $\ell_{\text {orb }} \gg 1$, where our CFT computation is valid. In this limit,

$$
\begin{aligned}
\mathcal{R}(r) & =\frac{r^{\ell_{\text {orb }}}}{\left(1+r^{2}\right)^{\frac{\ell_{\text {orb }}}{2}}} \frac{1}{\left(1+r^{2}\right)^{\frac{\Delta}{2}}}, \\
\mathcal{N} & =i \gamma_{E} \sqrt{\frac{\Gamma\left(\ell_{\text {orb }}+\Delta\right)}{\Gamma(\Delta-1) \Gamma\left(\ell_{\text {orb }}+2\right)}} \frac{\Gamma(\Delta-2)}{\Gamma(\Delta-1)}
\end{aligned}
$$

Performing the $r$ integral we find,

$$
\begin{aligned}
& \delta E_{\text {orb }}\left(0, \ell_{\text {orb }}\right)=-\frac{\mu}{2}|\mathcal{N}|^{2}(-\frac{\Gamma\left(\Delta+\frac{3}{2}\right)\left(\Delta+\ell_{\text {orb }}\right)^{2} \Gamma\left(\ell_{\text {orb }}+\frac{1}{2}\right)}{2 \Gamma\left(\ell_{\text {orb }}+\Delta+2\right)} \\
&\left.+\frac{\Gamma\left(\Delta+\frac{1}{2}\right)\left(\Delta+\ell_{\text {orb }}\right)\left((4 \Delta+3) \ell_{\text {orb }}-\Delta\right) \Gamma\left(\ell_{\text {orb }}-\frac{1}{2}\right)}{8 \Gamma\left(\ell_{\text {orb }}+\Delta+2\right)}\right) \\
& \approx-\gamma_{E}^{2} \frac{2 G_{N} M_{B H}}{3 \pi} \frac{\Delta(\Delta-1) \Gamma^{2}(\Delta-2)}{\Gamma^{2}(\Delta-1)}\left(\frac{1}{\ell_{\text {orb }}}-\frac{1}{\ell_{\text {orb }}^{2}}\right)
\end{aligned}
$$

Note that the formula we have is for a particle's motion around the black hole. But originally we had two particle orbiting rapidly in vacuum $\mathrm{AdS}_{5}$ as a the dual to double-trace primary operator. Therefore we need to map back to that two particle picture (see [48]) to obtain,

$$
\delta E_{\mathrm{orb}}\left(0, \ell_{\mathrm{orb}}\right) \approx-\gamma_{E}^{2} \frac{2 G_{N} \Delta_{1}}{3 \pi} \frac{\Delta_{2}\left(\Delta_{2}-1\right) \Gamma^{2}\left(\Delta_{2}-2\right)}{\Gamma^{2}\left(\Delta_{2}-1\right)}\left(\frac{2 \Delta_{1}}{\ell^{2}}\right)
$$

It is evident that (4.25) doesn't identically match to (3.16) that we derived using analytic bootstrap techniques. This is not unexpected, given the simpleminded dual gravity model for $\log C F T$ we have considered. The only thing we want to extract from this result is the large $\ell$ dependence. The binding energy which is equivalent to the anomalous dimension in the LogCFT side decreases like $\frac{1}{\ell^{2}}$ since $\ell$ is large. In the $\ell \rightarrow \infty$ limit, $\delta E_{\text {orb }} \rightarrow 0$, which means the particles are far from each other in AdS space and therefore effectively behave as "free" particles. This is a statement of cluster decomposition of the LogCFT in dual AdS language. 


\section{Conclusion}

In this paper we have studied logarithmic conformal field theory using analytic bootstrap techniques in four dimensions. In particular, we studied the four point correlator of rank2 identical logarithmic scalars. Using the bootstrap techniques we have shown how the intermediate double-trace operators in the large spin limit can reproduce the mean field theory correlator. We have computed the leading correction to the anomalous dimension of even spin rank-3 operators appearing in the OPE of two rank-2 logarithmic scalars in the large spin limit. LogCFTs are known to have holographic dual. The anomalous dimension of the double trace operators due to stress tensor exchange in four dimensions can be interpreted as the binding energy of the two particles rotating rapidly in global AdS. Our results also indicate that cluster decomposition holds (at least for large spin sector) even for certain class of non-unitary CFTs namely LogCFTs in $d>2$ with no operator with negative scaling dimensions. This is an interesting feature since unitarity condition plays key role in proving cluster decomposition for ordinary CFTs. There are many interesting directions to pursue in future.

- The subleading corrections in $1 / \ell$ to the anomalous dimension can be computed. This can be simplified in Mellin space following [58]. One can also take into account the higher twist double trace operators for $n \neq 0$. It would be nice to compute the anomalous dimension in the bulk side due to the exchange of higher rank stress-tensor. Moreover, the bulk and boundary analysis of rank-2 LogCFTs may be generalised to rank- $r$ LogCFTs by incorporating higher derivative action in the dual rank- $r$ LogCFTs. It would be interesting to repeat the analysis in general dimensions.

- In this note we have not used the mean field values of OPE coefficients $a_{p}, b_{p}, c_{p}$ for the minimal twist operators in (3.16). Given a LogCFT one can compute these coefficients. As LogCFTs are necessarily non-unitary they can, in general, be complex numbers. This can lead to some interesting physical phenomena both in the LogCFT side and in the dual bulk picture (e.g. the energy and loci of the rotating particles).

- Probably the most physically interesting systems to study are those LogCFTs which appear in condensed matter systems. For example, it would be nice to explore the $Q$ state Potts model using conformal bootstrap. As we have mentioned before the large spin sectors of all these particular systems are identical and we have already analysed that in section 2 in this paper. Also, in principle, the same large spin systemics should work for computing the corresponding anomalous dimensions for all those interesting LogCFTs.

- Another interesting but somewhat different direction will be to explore other known or conjectured non-unitary CFTs (which are not necessarily LogCFTs) by the same technique. E.g. one can analytically bootstrap non-unitary $\mathcal{N}=4$ theory [75] in $4 \mathrm{~d}$ with the gauge group $\mathrm{U}(N+k \mid k)$. This theory is said to be indistinguishable from its unitary cousin namely $\mathcal{N}=4 \mathrm{SYM}$ with gauge group $\mathrm{SU}(N)$ in arbitrary orders in $1 / N$. Since bootstrap methods are non-perturbative one can hope to capture their difference which is expected to be $\mathcal{O}\left(e^{-N}\right)$.

We hope to return to some of these problems in future. 


\section{Acknowledgments}

We thank Agnese Bissi, John Cardy and Aninda Sinha for useful discussions. Specifically, we thank Agnese Bissi and Aninda Sinha for comments on the draft. PB is grateful to the MPI Partner group grant MAXPLA/PHY/2018577. PD is supported by the Knut and Alice Wallenberg Foundation grant KAW 2016.0129.

\section{A Conformal blocks at large $\ell$}

The conformal block in four dimensions is given by,

$$
\mathcal{G}_{\Delta, \ell}(u, v)=\frac{1}{2^{\ell}} \frac{z \bar{z}}{z-\bar{z}}\left(k_{\Delta+\ell}(z) k_{\Delta-\ell-2}(\bar{z})-k_{\Delta+\ell}(\bar{z}) k_{\Delta-\ell-2}(z)\right) .
$$

We will be working in the limit $\ell \rightarrow \infty$ keeping $v \ell^{2}=y$ fixed. Note that $k_{2 \ell+\tau}(z)$ is proportional to $z^{\ell}$ and since we are in the regime $z \ll 1$. Hence this term is exponentially suppressed at large $\ell$ and we are left with,

$$
\mathcal{G}_{\Delta, \ell}(u, v) \sim \frac{1}{2^{\ell}} u k_{\Delta+\ell}(\bar{z}) k_{\Delta-\ell-2}(z),
$$

where,

$$
k_{\Delta-\ell-2}(z)=u^{\frac{\tau}{2}}+\cdots
$$

and the dots indicate higher order terms in $u$. For $k_{\Delta+\ell}(\bar{z})$ let us consider the integral representation of the hypergeometric function in the limit $\ell \rightarrow \infty$,

$$
\begin{aligned}
{ }_{2} F_{1}\left[\frac{\Delta+\ell}{2}, \frac{\Delta+\ell}{2}, \Delta+\ell, \bar{z}\right] & ={ }_{2} F_{1}\left[\frac{\tau}{2}+\ell, \frac{\tau}{2}+\ell, \tau+2 \ell, 1-v\right] \\
& =\frac{\Gamma(2 \ell)}{\Gamma^{2}(\ell)} \int_{0}^{1} \frac{d t}{t(1-t)}\left(\frac{t(1-t)}{1-t v}\right)^{\ell} \\
& \approx \frac{2^{2 \ell-1} \sqrt{\ell}}{\sqrt{\pi}} \int_{0}^{1} d t \frac{t^{\ell-1}}{1-t} e^{-\frac{t y}{(1-t) y}} .
\end{aligned}
$$

Now we define a new variable $s=\frac{t}{1-t}$ and rewite the integral as,

$$
\begin{aligned}
{ }_{2} F_{1}\left[\frac{\tau}{2}+\ell, \frac{\tau}{2}+\ell, \tau+2 \ell, 1-v\right] & \sim \frac{2^{2 \ell-1} \sqrt{\ell}}{\sqrt{\pi}} \int_{0}^{\infty} \frac{d s}{s} e^{-\frac{s y}{\ell}-\frac{\ell}{s}} \\
& =\frac{2^{2 \ell} \sqrt{\ell}}{\sqrt{\pi}} K_{0}(2 \ell \sqrt{v})+O(1 / \ell) .
\end{aligned}
$$

Now we will see how the derivative of (A.2) behaves in the large $\ell$ limit.

$$
\begin{aligned}
\partial_{\Delta} \mathcal{G}_{\Delta, \ell}(u, v) \sim & \frac{1}{2^{\ell}} u\left[\frac{1}{2} \log \bar{z} k_{\Delta+\ell}(\bar{z}) k_{\Delta-\ell-2}(z)+\frac{1}{2} \log z k_{\Delta+\ell}(\bar{z}) k_{\Delta-\ell-2}(z)\right. \\
& +\bar{z}^{\frac{\Delta+\ell}{2}} \partial_{\Delta 2} F_{1}\left[\frac{\Delta+\ell}{2}, \frac{\Delta+\ell}{2}, \Delta+\ell, \bar{z}\right] k_{\Delta-\ell-2}(z) \\
& \left.+z^{\frac{\Delta-\ell-2}{2}} k_{\Delta+\ell}(\bar{z}) \partial_{\Delta} F_{1}\left(\frac{\Delta-\ell-2}{2}, \frac{\Delta-\ell-2}{2}, \Delta-\ell-2, z\right)\right] .
\end{aligned}
$$


Note that,

$$
\log \bar{z}=\log (1-v)=-v+O\left(v^{2}\right) .
$$

Hence the term involving $\log \bar{z}$ in (A.6) is subleading in $v$ and can be ignored. Now we look at the derivatives of the ${ }_{2} F_{1}$.

$$
\begin{aligned}
& \partial_{\Delta} F_{1}\left(\frac{\Delta-\ell-2}{2}, \frac{\Delta-\ell-2}{2}, \Delta-\ell-2, z\right) \\
& =\sum_{n=0}^{\infty} \frac{z^{n} \Gamma(\tau-2) \Gamma\left(n+\frac{\tau}{2}-1\right)^{2}}{n ! \Gamma\left(\frac{\tau-2}{2}\right)^{2} \Gamma(n+\tau-2)}\left(H_{n+\frac{\tau}{2}-2}-H_{n+\tau-3}-H_{\frac{\tau}{2}-2}+H_{\tau-3}\right) .
\end{aligned}
$$

The leading term vanishes for $n=0$ and this is again subleading in $u$. Hence, we can ignore this term as well. We will finally focus on the second line of (A.6). Using the integral representation of the hypergeometric function we get,

$$
\begin{aligned}
\partial_{\Delta 2} F_{1}\left[\frac{\Delta+\ell}{2}, \frac{\Delta+\ell}{2}, \Delta+\ell, \bar{z}\right]= & \partial_{\Delta} \int_{0}^{1} d t \frac{\Gamma(\ell+\Delta)(-(t-1) t)^{\frac{1}{2}(\Delta+\ell-2)}(1-t \bar{z})^{\frac{1}{2}(-\Delta-\ell)}}{\Gamma\left(\frac{\ell+\Delta}{2}\right)^{2}} \\
= & \int_{0}^{1} d t \frac{(-(t-1) t)^{\ell+\frac{\tau}{2}-1} \Gamma(2 \ell+\tau) \log \left(\frac{(t-1) t}{t \bar{z}-1}\right)(1-t \bar{z})^{-\ell-\frac{\tau}{2}}}{2 \Gamma\left(\ell+\frac{\tau}{2}\right)^{2}} \\
& -\int_{0}^{1} d t \frac{(-(t-1) t)^{\ell+\frac{\tau}{2}-1} \Gamma(2 \ell+\tau)(1-t \bar{z})^{-\ell-\frac{\tau}{2}}}{\Gamma\left(\ell+\frac{\tau}{2}\right)^{2}} \\
& \times\left(\psi\left(\ell+\frac{\tau}{2}\right)-\psi(2 \ell+\tau)\right) .
\end{aligned}
$$

In order to do the integral we define a new variable $s=\frac{t}{1-t}$ and rewrite the first integral as follows,

$$
\int_{0}^{\infty} s y e^{-\frac{s y}{\ell}-\frac{\ell}{s}}\left(\frac{1}{\ell^{2} s}+\frac{1}{s^{2}}\right) d s=4 \sqrt{v} K_{1}(2 \sqrt{y})+\text { subleading terms } .
$$

Hence this is suppressed in $v$ and can be ignored. In the $\ell \rightarrow \infty$ limit,

$$
2 \psi(2 \ell+\tau)-2 \psi\left(\frac{1}{2}(2 \ell+\tau)\right) \sim 2 \ln (2)+O(1 / \ell)
$$

Hence, we have

$$
\sum_{\ell \gg 1} 2 q_{0, \ell} \partial_{\Delta} G_{\Delta, \ell}(u, v)=2\left(\frac{u}{v^{2}}\right)^{\frac{\Delta_{\phi}}{3}} \log 2 .
$$

\section{B Some details of the bulk computation}

Let's start with the solution (4.13) for the differential equation (4.8),

$$
\begin{aligned}
\Phi(x)= & \int \sqrt{-g} d^{d+1} x^{\prime} G\left(x, x^{\prime}\right) \Phi_{\mathrm{CFT}}\left(x^{\prime}\right) \\
= & \int \sqrt{-g} d t^{\prime} d \rho^{\prime} d \Omega^{\prime} \int \frac{d \omega}{2 \pi i} \sum_{n, \ell, \vec{m}} \frac{e^{i \omega\left(t-t^{\prime}\right)}}{\omega_{n, \ell}^{2}-\omega^{2}} \phi_{n, \ell, \vec{m}}^{*}(\vec{x}) \phi_{n, \ell, \vec{m}}\left(\vec{x}^{\prime}\right) \\
& \times \frac{1}{N_{\Delta, n, \ell}} e^{i \omega_{n, \ell} t^{\prime}} Y_{\ell J}\left(\Omega^{\prime}\right) \sin ^{\ell} \rho^{\prime} \cos ^{\Delta} \rho^{\prime}{ }_{2} F_{1}\left(-n, \Delta+n+\ell, \ell+\frac{d}{2}, \sin ^{2} \rho^{\prime}\right) .
\end{aligned}
$$


Although we write (B.1) for arbitrary $d$, we will work in $d=4$ in what follows. The above integration is over all coordinates i.e. $t^{\prime}, \rho^{\prime}$ and $\Omega^{\prime}$. Performing the $t^{\prime}$ integral first and then the $\omega$ integral we get,

$$
\begin{aligned}
\Phi(x)= & \int \sqrt{-g} d \rho^{\prime} d \Omega^{\prime} \sum_{n^{\prime}, \ell^{\prime}, \vec{m}^{\prime}} \frac{\phi_{n^{\prime}, \ell^{\prime}, \vec{m}^{\prime}}^{*}(\vec{x}) \phi_{n^{\prime}, \ell^{\prime}, \vec{m}^{\prime}}\left(\vec{x}^{\prime}\right)}{i\left(\omega_{n^{\prime}, \ell^{\prime}}^{2}-\omega_{n, \ell}^{2}\right)} \\
& \times \frac{e^{i \omega_{n, \ell} t}}{N_{\Delta, n, \ell}} Y_{\ell J}\left(\Omega^{\prime}\right) \sin ^{\ell} \rho^{\prime} \cos ^{\Delta} \rho^{\prime}{ }_{2} F_{1}\left(-n, \Delta+n+\ell, \ell+\frac{d}{2}, \sin ^{2} \rho^{\prime}\right) .
\end{aligned}
$$

The modes $\phi_{n, \ell, \vec{m}}(x)$ are known functions,

$$
\phi_{n, \ell, \vec{m}}(x)=Y_{\ell m}(\Omega) \sin ^{\ell} \rho \cos ^{\Delta} \rho P_{n}^{\ell+d / 2-1, \nu}(\cos 2 \rho),
$$

where $P_{n}^{m, \nu}(\cos 2 \rho)$ are Jacobi polynomials. Next we use the orthogonality of spherical harmonics (see e.g. complement $A_{\mathrm{VI}}$ of [76])

$$
\int d \Omega^{\prime} Y_{\ell^{\prime} m^{\prime}}^{*}\left(\Omega^{\prime}\right) Y_{\ell J}\left(\Omega^{\prime}\right)=\delta_{\ell, \ell^{\prime}} \delta_{J m^{\prime}}
$$

to obtain,

$$
\begin{aligned}
\Phi(x)= & \int \sqrt{-g} d \rho^{\prime} \sum_{n^{\prime}} \frac{1}{\left(\omega_{n^{\prime}, \ell}^{2}-\omega_{n, \ell}^{2}\right)} P_{n^{\prime}}^{\ell+d / 2-1, \nu}(\cos 2 \rho) P_{n^{\prime}}^{\ell+d / 2-1, \nu}\left(\cos 2 \rho^{\prime}\right) \\
& \times Y_{\ell J}^{*}(\Omega)(\sin \rho)^{\ell}(\cos \rho)^{\Delta} \frac{e^{i \omega_{n, \ell} t}}{i N_{\Delta, n, \ell}}\left(\sin \rho^{\prime}\right)^{2 \ell}\left(\cos \rho^{\prime}\right)^{2 \Delta}{ }_{2} F_{1}\left(-n, \Delta+n+\ell, \ell+\frac{d}{2}, \sin ^{2} \rho^{\prime}\right) \\
= & \frac{Y_{\ell J}^{*}(\Omega) e^{i \omega_{n, \ell} t}}{i N_{\Delta, n, \ell}} \frac{n !}{\left(\ell+\frac{d}{2}\right)_{n}}(\sin \rho)^{\ell}(\cos \rho)^{\Delta} \sum_{n^{\prime}} \frac{1}{\left(\omega_{n^{\prime}, \ell}^{2}-\omega_{n, \ell}^{2}\right)} P_{n^{\prime}}^{\ell+d / 2-1, \nu}(\cos 2 \rho) \\
& \times \int_{0}^{\pi / 2} d \rho^{\prime}\left(\sin \rho^{\prime}\right)^{2 \ell-d-1}\left(\cos \rho^{\prime}\right)^{2 \Delta+d-1} P_{n^{\prime}}^{\ell+d / 2-1, \nu}\left(\cos 2 \rho^{\prime}\right) P_{n}^{\ell+d / 2-1, \nu}\left(\cos 2 \rho^{\prime}\right) \\
\equiv & \frac{Y_{\ell J}^{*}(\Omega) e^{i \omega_{n, \ell} t}}{i N_{\Delta, n, \ell}} \frac{n !}{\left(\ell+\frac{d}{2}\right)_{n}}(\sin \rho)^{\ell}(\cos \rho)^{\Delta} f(\rho),
\end{aligned}
$$

where $\sqrt{-g}=\left(\sin \rho^{\prime}\right)^{-d-1}\left(\cos \rho^{\prime}\right)^{d-1}$, and $\alpha=\ell+\frac{d}{2}-1 ; \quad \beta=\Delta-\frac{d}{2}=\nu$.

To obtain the solution $\Phi(x)$ one needs to compute $f(\rho)$. One way would be to perform the $n^{\prime}$ sum and $\rho^{\prime}$ integral. Here we take an alternative approach, namely we substitute (B.5) to (4.8) as an ansatz to get the following differential equation for $f(\rho)$,

$$
f^{\prime \prime}(\rho)-((2 \Delta-3) \tan \rho-(2 \ell+3) \cot \rho) f^{\prime}(\rho)-(\Delta-\omega+\ell)(\Delta+\omega+\ell) f(\rho)=\sec ^{2} \rho .
$$

All one needs to do is to solve for $f(\rho)$. The equation (B.6) is an second-order inhomogeneous ODE. Let's make the following change of variables,

$$
z=\cos ^{2} \rho .
$$

Above equation (B.6) reduces to,

$$
(1-z) z f^{\prime \prime}(z)+(c-(a+b+1) z) f^{\prime}(z)-a b f(z)=z^{q},
$$


with,

$$
\begin{aligned}
a & =\frac{1}{2}(\Delta-\omega+\ell)=-n, \\
b & =\frac{1}{2}(\Delta+\omega+\ell)=n+\ell+\Delta \\
c & =\Delta-1 \\
q & =-1 .
\end{aligned}
$$

The corresponding homogeneous ODE is the standard hypergeometric differential equation,

$$
(1-z) z f^{\prime \prime}(z)+(c-(a+b+1) z) f^{\prime}(z)-a b f(z)=0,
$$

with the following solution,

$$
f^{h}(z)=C_{12} F_{1}(a, b, c ; z)+C_{2} z^{1-c}{ }_{2} F_{1}(b-c+1, a-c+1,2-c ; z) .
$$

Imposing regularity at the centre of $\operatorname{AdS}(z \rightarrow \infty)$, forces one to choose $C_{2}=0$. Other boundary condition fixes the normalization $C_{1}=\mathcal{N}_{\Delta, \ell, m}$. The inhomogeneous DE (B.6), has the following particular solution, ${ }^{5}$

$$
f^{p}(z)=\frac{\Gamma(1+q) \Gamma(c-1)}{\Gamma(c)}{ }_{2} F_{1}(a, b ; c ; z) .
$$

Notice that the solution (B.11) is well behaved for all values of $q$, except for $q \in \mathbb{Z}^{-}$. One can analytically continue to complex $\mathbf{q}=q_{1}+i q_{2}$. Then for any negative integer $\mathbf{q}=-p$, one can expand $\Gamma(1+\mathbf{q})$ in small complex neighborhood as following, ${ }^{6}$

$$
\begin{aligned}
\Gamma(1+\mathbf{q}) & =\Gamma(1-p+i \epsilon) \\
& =\frac{(-1)^{p-1}}{(p-1) !}\left(\frac{1}{i \epsilon}+\psi(p)+\mathcal{O}(\epsilon)\right) .
\end{aligned}
$$

It is evident that in the limit $\epsilon \rightarrow 0$, only the imaginary part blows up, whereas the real part is independent of $\epsilon .^{7}$ Thus there is a consistent prescription of extracting $\epsilon$ independent value as follows

$$
\Gamma(1-p)=\frac{(-1)^{p-1}}{(p-1) !} \psi(p) .
$$

Here we are particularly interested in $q=-1$ i.e. $p=1$, for which

$$
\Gamma(1+q)=\psi(1)=-\gamma_{E} .
$$

\footnotetext{
${ }^{5}$ See eq. (12) and eq. (13) of [77].

${ }^{6}$ Near any simple pole at $z=-n$, where $n \in \mathbb{Z}^{+}$,

$$
\Gamma(-n+z)=\frac{(-1)^{n}}{n !}\left(\frac{1}{z}+\psi(n+1)+\mathcal{O}(z)\right) .
$$

${ }^{7}$ The function $\Gamma(1+\mathbf{q})$ is analytic on the complex $\mathbf{q}$-plane with $q \in \mathbb{Z}^{-}$removed - which is an open set. Therefore one can approach the disconnected singular points at $q \in \mathbb{Z}^{-}$from any directions in the complex q-plane. The finite part will be independent of the cut-off.
} 
Therefore the particular solution reduces to,

$$
f^{p}(z)=-\gamma_{E} \frac{\Gamma(c-1)}{\Gamma(c)}{ }_{2} F_{1}(a, b ; c ; z) .
$$

The argument of the hypergeometric function is $z=\cos ^{2} \rho$. Since we want the solution with the variable $\sin ^{2} \rho$, let's use the following identity,

$$
{ }_{2} F_{1}(-m, b ; c ; z)=\frac{(c-b)_{m}}{(c)_{m}}{ }_{2} F_{1}(-m, b ; b-c-m+1 ; 1-z),
$$

to get,

$$
\begin{aligned}
f^{p}(z) & =-\gamma_{E} \frac{\Gamma(c-1)}{\Gamma(c)} \frac{(c-b)_{n}}{(c)_{n}}{ }_{2} F_{1}(-n, b ; b-c-n+1 ; 1-z) \\
& =-\gamma_{E} \frac{\Gamma(\Delta-2) \Gamma(n+\ell+2)}{\Gamma(n+\Delta-1) \Gamma(\ell+2)}{ }_{2} F_{1}\left(-n, n+\ell+\Delta ; \ell+2 ; \sin ^{2} \rho\right) .
\end{aligned}
$$

Now we have all the ingredients to write down the full solution to (4.8),

$$
\begin{aligned}
\Phi(x)= & -\gamma_{E} \frac{Y_{l J}^{*}(\Omega) e^{i \omega_{n, \ell} t}}{i N_{\Delta, n, l}}(\sin \rho)^{\ell}(\cos \rho)^{\Delta} \frac{n !}{\left(\ell+\frac{d}{2}\right)_{n}} \frac{\Gamma(\Delta-2) \Gamma(n+\ell+2)}{\Gamma(n+\Delta-1) \Gamma(\ell+2)} \\
& \times{ }_{2} F_{1}\left(-n, n+\ell+\Delta ; \ell+2 ; \sin ^{2} \rho\right) .
\end{aligned}
$$

Open Access. This article is distributed under the terms of the Creative Commons Attribution License (CC-BY 4.0), which permits any use, distribution and reproduction in any medium, provided the original author(s) and source are credited.

\section{References}

[1] L. Rozansky and H. Saleur, Quantum field theory for the multivariable Alexander-Conway polynomial, Nucl. Phys. B 376 (1992) 461 [INSPIRE].

[2] H. Saleur, Polymers and percolation in two-dimensions and twisted $N=2$ supersymmetry, Nucl. Phys. B 382 (1992) 486 [hep-th/9111007] [INSPIRE].

[3] V. Gurarie, Logarithmic operators in conformal field theory, Nucl. Phys. B 410 (1993) 535 [hep-th/9303160] [INSPIRE].

[4] J. Cardy, Logarithmic conformal field theories as limits of ordinary CFTs and some physical applications, J. Phys. A 46 (2013) 494001 [arXiv: 1302.4279] [InSPIRE].

[5] J.L. Cardy, Logarithmic correlations in quenched random magnets and polymers, cond-mat/9911024.

[6] M.R. Rahimi Tabar and S. Rouhani, Turbulent two-dimensional magnetohydrodynamics and conformal field theory, Annals Phys. 246 (1996) 446 [hep-th/9503005] [INSPIRE].

[7] M.R. Rahimi Tabar and S. Rouhani, Logarithmic correlation functions in two-dimensional turbulence, Phys. Lett. A 224 (1997) 331 [hep-th/9606154] [INSPIRE].

[8] M.A.I. Flohr, Two-dimensional turbulence: Yet another conformal field theory solution, Nucl. Phys. B 482 (1996) 567 [hep-th/9606130] [INSPIRE]. 
[9] P. Ruelle, A c=-2 boundary changing operator for the Abelian sandpile model, Phys. Lett. B 539 (2002) 172 [hep-th/0203105] [INSPIRE].

[10] S. Mahieu and P. Ruelle, Scaling fields in the two-dimensional Abelian sandpile model, Phys. Rev. E 64 (2001) 066130 [hep-th/0107150] [INSPIRE].

[11] M. Jeng, G. Piroux and P. Ruelle, Height variables in the Abelian sandpile model: Scaling fields and correlations, J. Stat. Mech. 0610 (2006) P10015 [cond-mat/0609284] [INSPIRE].

[12] J.S. Caux, I.I. Kogan and A.M. Tsvelik, Logarithmic operators and hidden continuous symmetry in critical disordered models, Nucl. Phys. B 466 (1996) 444 [hep-th/9511134] [INSPIRE].

[13] V. Gurarie, c theorem for disordered systems, Nucl. Phys. B 546 (1999) 765 [cond-mat/9808063] [INSPIRE].

[14] M.J. Bhaseen, I.I. Kogan, O.A. Solovev, N. Tanigichi and A.M. Tsvelik, Towards a field theory of the plateau transitions in the integer quantum Hall effect, Nucl. Phys. B 580 (2000) 688 [cond-mat/9912060] [INSPIRE].

[15] I.I. Kogan and A.M. Tsvelik, Logarithmic operators in the theory of plateau transition, Mod. Phys. Lett. A 15 (2000) 931 [hep-th/9912143] [INSPIRE].

[16] A.W.W. Ludwig, A Free field representation of the $\operatorname{Osp}(2 \mid 2)$ current algebra at level $k=-2$ and Dirac fermions in a random $\mathrm{SU}(2)$ gauge potential, cond-mat/0012189.

[17] N. Read and H. Saleur, Exact spectra of conformal supersymmetric nonlinear $\sigma$-models in two-dimensions, Nucl. Phys. B 613 (2001) 409 [hep-th/0106124] [INSPIRE].

[18] I.I. Kogan and N.E. Mavromatos, World sheet logarithmic operators and target space symmetries in string theory, Phys. Lett. B 375 (1996) 111 [hep-th/9512210] [INSPIRE].

[19] V. Periwal and O. Tafjord, D-brane recoil, Phys. Rev. D 54 (1996) R3690 [hep-th/9603156] [INSPIRE].

[20] N.E. Mavromatos and R.J. Szabo, Matrix D-brane dynamics, logarithmic operators and quantization of noncommutative space-time, Phys. Rev. D 59 (1999) 104018 [hep-th/9808124] [INSPIRE].

[21] K. Sfetsos, String backgrounds and LCFT, Phys. Lett. B 543 (2002) 73 [hep-th/0206091] [INSPIRE].

[22] I. Bakas and K. Sfetsos, PP waves and logarithmic conformal field theories, Nucl. Phys. B 639 (2002) 223 [hep-th/0205006] [INSPIRE].

[23] I.I. Kogan and D. Polyakov, World sheet logarithmic CFT in AdS strings, ghost matter mixing and M-theory, Int. J. Mod. Phys. A 16 (2001) 2559 [hep-th/0012128] [INSPIRE].

[24] A.M. Ghezelbash, M. Khorrami and A. Aghamohammadi, Logarithmic conformal field theories and AdS correspondence, Int. J. Mod. Phys. A 14 (1999) 2581 [hep-th/9807034] [INSPIRE].

[25] I.I. Kogan, Singletons and logarithmic CFT in AdS/CFT correspondence, Phys. Lett. B 458 (1999) 66 [hep-th/9903162] [INSPIRE].

[26] S. Moghimi-Araghi, S. Rouhani and M. Saadat, On the AdS/CFT correspondence and logarithmic operator, Phys. Lett. B 518 (2001) 157 [hep-th/0105123] [INSPIRE]. 
[27] S. Jabbari-Faruji and S. Rouhani, Calculation of four point correlation function of logarithmic conformal field theory using AdS/CFT correspondence, Phys. Lett. B 548 (2002) 237 [hep-th/0205016] [INSPIRE].

[28] D. Grumiller, W. Riedler, J. Rosseel and T. Zojer, Holographic applications of logarithmic conformal field theories, J. Phys. A 46 (2013) 494002 [arXiv: 1302.0280] [inSPIRE].

[29] E. Witten, Open Strings On The Rindler Horizon, JHEP 01 (2019) 126 [arXiv:1810.11912] [INSPIRE].

[30] M. Hogervorst, M. Paulos and A. Vichi, The ABC (in any D) of Logarithmic CFT, JHEP 10 (2017) 201 [arXiv: 1605.03959] [INSPIRE].

[31] A.A. Migdal, Conformal invariance and bootstrap, Phys. Lett. 37B (1971) 386 [InSPIRE].

[32] S. Ferrara, A.F. Grillo, G. Parisi and R. Gatto, Covariant expansion of the conformal four-point function, Nucl. Phys. B 49 (1972) 77 [Erratum ibid. B 53 (1973) 643] [INSPIRE].

[33] S. Ferrara, A.F. Grillo and R. Gatto, Tensor representations of conformal algebra and conformally covariant operator product expansion, Annals Phys. 76 (1973) 161 [INSPIRE].

[34] A.M. Polyakov, Nonhamiltonian approach to conformal quantum field theory, Zh. Eksp. Teor. Fiz. 66 (1974) 23 [INSPIRE].

[35] R. Rattazzi, V.S. Rychkov, E. Tonni and A. Vichi, Bounding scalar operator dimensions in 4D CFT, JHEP 12 (2008) 031 [arXiv:0807.0004] [INSPIRE].

[36] V.S. Rychkov and A. Vichi, Universal Constraints on Conformal Operator Dimensions, Phys. Rev. D 80 (2009) 045006 [arXiv:0905.2211] [InSPIRE].

[37] D. Poland and D. Simmons-Duffin, Bounds on 4D Conformal and Superconformal Field Theories, JHEP 05 (2011) 017 [arXiv: 1009.2087] [INSPIRE].

[38] R. Rattazzi, S. Rychkov and A. Vichi, Central Charge Bounds in $4 D$ Conformal Field Theory, Phys. Rev. D 83 (2011) 046011 [arXiv: 1009.2725] [InSPIRE].

[39] R. Rattazzi, S. Rychkov and A. Vichi, Bounds in 4D Conformal Field Theories with Global Symmetry, J. Phys. A 44 (2011) 035402 [arXiv: 1009.5985] [InSPIRE].

[40] D. Poland, D. Simmons-Duffin and A. Vichi, Carving Out the Space of $4 D$ CFTs, JHEP 05 (2012) 110 [arXiv:1109.5176] [INSPIRE].

[41] F. Gliozzi, More constraining conformal bootstrap, Phys. Rev. Lett. 111 (2013) 161602 [arXiv:1307.3111] [INSPIRE].

[42] F. Gliozzi and A. Rago, Critical exponents of the $3 d$ Ising and related models from Conformal Bootstrap, JHEP 10 (2014) 042 [arXiv: 1403.6003] [INSPIRE].

[43] S. El-Showk, M.F. Paulos, D. Poland, S. Rychkov, D. Simmons-Duffin and A. Vichi, Solving the 3D Ising Model with the Conformal Bootstrap, Phys. Rev. D 86 (2012) 025022 [arXiv: 1203.6064] [INSPIRE].

[44] S. El-Showk, M.F. Paulos, D. Poland, S. Rychkov, D. Simmons-Duffin and A. Vichi, Solving the $3 d$ Ising Model with the Conformal Bootstrap II. c-Minimization and Precise Critical Exponents, J. Stat. Phys. 157 (2014) 869 [arXiv:1403.4545] [InSPIRE].

[45] F. Kos, D. Poland, D. Simmons-Duffin and A. Vichi, Precision Islands in the Ising and $O(N)$ Models, JHEP 08 (2016) 036 [arXiv:1603.04436] [INSPIRE]. 
[46] A.L. Fitzpatrick, J. Kaplan, D. Poland and D. Simmons-Duffin, The Analytic Bootstrap and AdS Superhorizon Locality, JHEP 12 (2013) 004 [arXiv:1212.3616] [INSPIRE].

[47] Z. Komargodski and A. Zhiboedov, Convexity and Liberation at Large Spin, JHEP 11 (2013) 140 [arXiv: 1212.4103] [INSPIRE].

[48] A.L. Fitzpatrick, J. Kaplan and M.T. Walters, Universality of Long-Distance AdS Physics from the CFT Bootstrap, JHEP 08 (2014) 145 [arXiv:1403.6829] [INSPIRE].

[49] L.F. Alday, A. Bissi and T. Lukowski, Large spin systematics in CFT, JHEP 11 (2015) 101 [arXiv: 1502.07707] [INSPIRE].

[50] A. Kaviraj, K. Sen and A. Sinha, Analytic bootstrap at large spin, JHEP 11 (2015) 083 [arXiv: 1502.01437] [INSPIRE].

[51] A. Kaviraj, K. Sen and A. Sinha, Universal anomalous dimensions at large spin and large twist, JHEP 07 (2015) 026 [arXiv: 1504.00772] [INSPIRE].

[52] L.F. Alday and A. Zhiboedov, Conformal Bootstrap With Slightly Broken Higher Spin Symmetry, JHEP 06 (2016) 091 [arXiv: 1506. 04659] [INSPIRE].

[53] L.F. Alday and A. Zhiboedov, An Algebraic Approach to the Analytic Bootstrap, JHEP 04 (2017) 157 [arXiv: 1510.08091] [INSPIRE].

[54] D. Li, D. Meltzer and D. Poland, Non-Abelian Binding Energies from the Lightcone Bootstrap, JHEP 02 (2016) 149 [arXiv: 1510.07044] [INSPIRE].

[55] P. Dey, A. Kaviraj and K. Sen, More on analytic bootstrap for $O(N)$ models, JHEP 06 (2016) 136 [arXiv: 1602.04928] [INSPIRE].

[56] L.F. Alday, Large Spin Perturbation Theory for Conformal Field Theories, Phys. Rev. Lett. 119 (2017) 111601 [arXiv:1611.01500] [INSPIRE].

[57] L.F. Alday, Solving CFTs with Weakly Broken Higher Spin Symmetry, JHEP 10 (2017) 161 [arXiv:1612.00696] [INSPIRE].

[58] P. Dey, K. Ghosh and A. Sinha, Simplifying large spin bootstrap in Mellin space, JHEP 01 (2018) 152 [arXiv: 1709.06110] [inSPIRE].

[59] P. Dey and A. Kaviraj, Towards a Bootstrap approach to higher orders of $\epsilon$-expansion, JHEP 02 (2018) 153 [arXiv: 1711.01173] [INSPIRE].

[60] R. Gopakumar, A. Kaviraj, K. Sen and A. Sinha, Conformal Bootstrap in Mellin Space, Phys. Rev. Lett. 118 (2017) 081601 [arXiv: 1609.00572] [INSPIRE].

[61] R. Gopakumar, A. Kaviraj, K. Sen and A. Sinha, A Mellin space approach to the conformal bootstrap, JHEP 05 (2017) 027 [arXiv: 1611.08407] [INSPIRE].

[62] P. Dey, A. Kaviraj and A. Sinha, Mellin space bootstrap for global symmetry, JHEP 07 (2017) 019 [arXiv : 1612.05032] [INSPIRE].

[63] R. Gopakumar and A. Sinha, On the Polyakov-Mellin bootstrap, JHEP 12 (2018) 040 [arXiv: 1809.10975] [INSPIRE].

[64] K. Ghosh, Polyakov-Mellin Bootstrap for AdS loops, arXiv:1811.00504 [INSPIRE].

[65] F.A. Dolan and H. Osborn, Conformal four point functions and the operator product expansion, Nucl. Phys. B 599 (2001) 459 [hep-th/0011040] [INSPIRE].

[66] F.A. Dolan and H. Osborn, Conformal partial waves and the operator product expansion, Nucl. Phys. B 678 (2004) 491 [hep-th/0309180] [INSPIRE]. 
[67] F.A. Dolan and H. Osborn, Conformal Partial Waves: Further Mathematical Results, arXiv:1108.6194 [INSPIRE].

[68] A.L. Fitzpatrick and J. Kaplan, Unitarity and the Holographic S-matrix, JHEP 10 (2012) 032 [arXiv: 1112.4845] [INSPIRE].

[69] L.F. Alday and J.M. Maldacena, Comments on operators with large spin, JHEP 11 (2007) 019 [arXiv: 0708.0672] [INSPIRE].

[70] L.F. Alday and A. Bissi, Higher-spin correlators, JHEP 10 (2013) 202 [arXiv:1305.4604] [INSPIRE].

[71] F.W.J. Olver et al. eds., NIST Digital Library of Mathematical Functions, http://dlmf.nist.gov/.

[72] E.A. Bergshoeff, S. de Haan, W. Merbis, M. Porrati and J. Rosseel, Unitary Truncations and Critical Gravity: a Toy Model, JHEP 04 (2012) 134 [arXiv:1201.0449] [INSPIRE].

[73] V. Balasubramanian, P. Kraus and A.E. Lawrence, Bulk versus boundary dynamics in anti-de Sitter space-time, Phys. Rev. D 59 (1999) 046003 [hep-th/9805171] [INSPIRE].

[74] A.L. Fitzpatrick, E. Katz, D. Poland and D. Simmons-Duffin, Effective Conformal Theory and the Flat-Space Limit of AdS, JHEP 07 (2011) 023 [arXiv:1007.2412] [INSPIRE].

[75] C. Vafa, Non-Unitary Holography, arXiv:1409.1603 [INSPIRE].

[76] C. Cohen-Tannoudji, B. Diu and F. Laloë, Quantum mechanics, Wiley, New York, NY (1977), Trans. of Mécanique quantique, Hermann, Paris (1973) [https://cds.cern.ch/record/101367].

[77] L.U. Ancarani and G. Gasaneo, Derivatives of any order of the Gaussian hypergeometric function ${ }_{2} F_{1}(a, b, c ; z)$ with respect to the parameters $a, b$ and $c$, J. Phys. A 42 (2009) 395208 [INSPIRE]. 